\title{
Scarring and the statistics of tunnelling
}

\author{
Stephen C. Creagh ${ }^{a}$, Soo-Young Lee ${ }^{a}$ and Niall D. Whelan ${ }^{b}$ \\ ${ }^{a}$ School of Mathematical Sciences, University of Nottingham, Nottingham NG7 2RD, UK. \\ ${ }^{b}$ Department of Physics and Astronomy, McMaster University, Hamilton, Ontario, Canada L8S $4 M 1$.
}

\begin{abstract}
We show that the statistics of tunnelling can be dramatically affected by scarring and derive distributions quantifying this effect. Strong deviations from the prediction of random matrix theory can be explained quantitatively by modifying the Gaussian distribution which describes wavefunction statistics. The modified distribution depends on classical parameters which are determined completely by linearised dynamics around a periodic orbit. This distribution generalises the scarring theory of Kaplan [Phys. Rev. Lett. 80, 2582 (1998)] to describe the statistics of the components of the wavefunction in a complete basis, rather than overlaps with single Gaussian wavepackets. In particular it is shown that correlations in the components of the wavefunction are present, which can strongly influence tunnellingrate statistics. The resulting distribution for tunnelling rates is tested successfully on a two-dimensional double-well potential.
\end{abstract}

\section{INTRODUCTION}

Tunnelling rates from systems with complex internal behaviour are naturally described statistically. Such analyses have been used in particular to understand nuclear resonances [1], chemical reaction rates [2 8] and quantum dots [9, 10]. While the manner in which internal states couple to the continuum varies in these analyses, the internal quantum mechanics is usually modelled using one of the standard ensembles of random matrix theory (RMT). We show here that in systems where the complexity of the internal dynamics derives from low-dimensional chaos, sufficiently strong deviation from the standard RMT statistics is possible that it dominates the statistics of tunnelling. These deviations were pointed out in [11] and are explained in detail here using the theory of scarring developed by Heller, Kaplan and coworkers [12, 13.

We consider tunnelling to and from regions of phase space associated with chaotic behaviour. In particular, our theory works for the calculation of level splittings in double well potentials and of resonance widths of quasibound states in metastable wells. With the development of experimental techniques capable of measuring state-specific reaction rates, such tunnelling from highly-excited chaotic states has become directly relevant to the analysis of chemical reactions, for example. Specific examples of reactions which have been treated using RMT-based statistical methods include the dissociation rates of $\mathrm{D}_{2} \mathrm{CO}$ [2, 3] and $\mathrm{N}_{2} \mathrm{O}$ [8]. In [11, the distributions arising from such an analysis were shown to be determined in a simple way by the stability and action of a complex tunnelling orbit which crosses the potential barrier with minimum imaginary action. The resulting statistical distributions for the tunnelling rate agree well with numerically computed ensembles except when the real extension of the tunnelling orbit into the potential well is periodic; in that case, strong deviations from the RMT prediction are observed and it was proposed in [11] that these are due to the effect of scarring on wavefunction statistics as outlined in [13]. Additional evidence in support of this has subsequently been provided in [14].

In this paper we derive a distribution describing scar-influenced tunnelling-rate statistics in twodimensional potentials. It is determined completely by three dynamical parameters which are calculated from the monodromy matrices of the tunnelling orbit and of its real periodic extension. These three parameters can be understood as the stability of the scarred orbit, the stability of the complex tunnelling orbit and an angle relating stable and unstable manifolds. The root of this calculation is a conjecture governing the statistics of the components of chaotic wavefunctions in the eigenbasis of a tunnelling operator which was defined in [15]. The low-lying states of this tunnelling operator are approximated by the eigenstates of 
a harmonic oscillator. Following the linear scarring theory of Kaplan [13] we show that the components of chaotic states in this basis have variances which deviate from those of RMT and which must furthermore be correlated. We conjecture that the eigenfunction distribution is a nonisotropic Gaussian. This is the simplest distribution consistent with the observed correlations and may also be obtained from maximum-entropy arguments. This conjecture is tested against quantum-map models and found to describe statistics of their eigenstates very accurately. The conjecture is then used to derive modified distributions for tunnelling rates in the presence of scarring and these are found to describe well the statistics of energy-level splittings in chaotic double well potentials.

We see the benefits of this work as being twofold. First, there is a solution to the primary problem of incorporating the effects of scarring into the statistics of tunnelling. This enables the detection and interpretation of system-specific dynamical detail in measurements of multidimensional tunnelling and furthermore provides a very direct manifestation of the phenomenon of scarring in quantities with real physical relevance. Second, we believe that from the point of view of scarring alone, even without reference to tunnelling, the correlated joint probability distribution proposed here for the statistics of eigenstates holds considerable promise as a theoretical tool. In particular, it suggests methods of quantifying the effects of scarring in a way that does not depend on a choice of test states. This second aspect is not fully exploited in the present paper, but we believe that the essential elements needed for a full development are put in place. We remark that the effect of correlations on the statistics of tunnelling rates was also considered in a somewhat different context in [10].

An outline of the paper is as follows. We begin in section II with a review of the existing theory of the statistics of tunnelling rates. This includes a definition of the tunnelling operator introduced in [15] and a summary of the derivation from it of nonscarred tunnelling-rate distributions as outlined previously in [11]. In section III we use the linear theory of scarring to deduce that in the presence of scarring the components of chaotic eigenstates in an eigenbasis of the tunnelling operator must be nonRMT and conjecture a modified Gaussian distribution for them. This conjecture is tested on quantisations of perturbed cat maps and found to work well. In section IV we use the conjecture to deduce modified tunnelling-rate distributions in the presence of scarring and these are compared successfully with distributions of splitting in chaotic double-well potentials.

\section{TUNNELLING STATISTICS WITHOUT SCARRING}

We begin with a brief description of existing theory of tunnelling-rate statistics in the absence of scarring, on which our present work is based. We first describe a tunnelling operator defined in [15] which relates tunnelling rates to the properties of wavefunctions in the allowed region. We then outline how this tunnelling operator is used to derive distributions for tunnelling rates in the absence of scarring.

\section{A. The tunnelling operator}

A full description of the semiclassical matrix element and complete definitions of the various objects needed to calculate it are described in [15]. For the purposes of statistical analysis, an idealised model formulated in terms of quantum maps suffices, which we summarise here.

Our model starts with a finite-dimensional Hilbert space $\mathcal{H}$. We take this space to be the quantum analog of a Poincaré section $\Sigma$ in a potential well [16], with the dimension $N$ of $\mathcal{H}$ being approximately proportional to the area of $\Sigma$. A quantum mapping acts on the space $\mathcal{H}$ as a unitary operator $\hat{U}$, whose eigensolutions we denote by

$$
\hat{U}|n\rangle=e^{-i \theta_{n}}|n\rangle
$$


and whose classical analog is a symplectic map $F$ corresponding to chaotic motion within the well. In [15], the eigenstates $|n\rangle$ are represented as certain cross-sections of the eigenfunctions of the usual Hamiltonian in the full Hilbert space.

Tunnelling rates are calculated using a tunnelling operator $\hat{\mathcal{T}}$ which also acts on $\mathcal{H}$ and from which the scaled tunnelling rate associated with a chaotic eigenstate $|n\rangle$ is calculated from

$$
y_{n}=\frac{\langle n|\hat{\mathcal{T}}| n\rangle}{\operatorname{Tr} \hat{\mathcal{T}}} .
$$

In this formula, we normalise the chaotic eigenstates according to $\langle n \mid n\rangle=N$ so that, in particular, the overlaps with a second basis $|\tilde{k}\rangle$ of $\mathcal{H}$ satisfy $\left\langle|\langle\tilde{k} \mid n\rangle|^{2}\right\rangle=1$. As a result, $\left\langle y_{n}\right\rangle=1$. In resonance problems, this scaled tunnelling rate is $y_{n}=\Gamma_{n} /\left\langle\Gamma_{n}\right\rangle$ where $\Gamma_{n}$ is the resonance width associated with a particular metastable state labeled by $n$ and in double-well problems $y_{n}=\Delta E_{n} /\left\langle\Delta E_{n}\right\rangle$ where $\Delta E_{n}$ is the splitting of a doublet labeled by $n$.

The tunnelling operator $\hat{\mathcal{T}}$ is interpreted as an evolution operator whose classical correspondent is a symplectic map $\mathcal{F}$ which is complex. This map $\mathcal{F}$ has a real fixed point $\zeta_{0}$ which corresponds to the most probable tunnelling route. That is, using $\zeta_{0}$ as an initial condition, a complex trajectory of imaginary period can be traced out which crosses a potential barrier with minimum imaginary action. Mappings of points in a neighbourhood of $\zeta_{0}$ in the Poincaré section $\Sigma$ correspond to complex trajectories in full phase space which cross the potential barrier near this central tunnelling route. The matrix element in (1) then samples the state $|n\rangle$ in a small region of $\Sigma$ surrounding $\zeta_{0}$ and with area of $O(\hbar)$ (as determined by say, a Wigner function on $\Sigma[15])$. This region is determined by the dynamics of $\mathcal{F}$ near $\zeta_{0}$ and outside of it the symbol of $\hat{\mathcal{T}}$ decays exponentially. For this reason it is consistent within semiclassical approximation to linearise dynamics around $\zeta_{0}$ and let $\hat{\mathcal{T}}$ be the quantisation of the resulting complex symplectic matrix $W$. Note that in practical terms $W$ is simply the monodromy matrix of the tunnelling orbit.

We now describe the tunnelling operator for resonance problems of symmetric double wells where the symmetry is a reflection $(x, y) \mapsto(-x, y)$. If we choose a representation in which $\zeta_{0}$ is at the centre of coordinates $\zeta=(q, p)$ on $\Sigma$, we may write

$$
\hat{\mathcal{T}}=e^{-\alpha_{0} \hat{h} / \hbar}
$$

for these cases, where $\hat{h}$ is quadratic in the corresponding operators $\hat{\zeta}=(\hat{q}, \hat{p})$ and $\alpha_{0}>0$. The corresponding symbol $h(q, p)$ is a quadratic form in $(q, p)$, expressed as

$$
h(q, p)=\frac{1}{2} \zeta^{T} K \zeta,
$$

where $K$ is a positive-definite symmetric matrix. The matrix $K$ is determined from $W$ by writing

$$
W=e^{-i \alpha_{0} J K} .
$$

where $J$ is the unit symplectic matrix. We normalise $K$ so that $\operatorname{det} K=1$ and then the parameter $\alpha_{0}$ is fixed by a calculation of $W$. That $W$ can be written in this way for real $\alpha_{0}$ and $K$ can be shown using the conjugate-time-reversal symmetry

$$
W^{*}=W^{-1},
$$

which is a special case of a similar symmetry of $\mathcal{F}[15]$. This symmetry is a reflection of the fact that the tunnelling orbit is periodic with an imaginary period and so complex conjugation is equivalent to timereversal. Note that this is the case even if the problem does not have a proper time-reversal symmetry (such as in the presence of magnetic fields, for example).

We limit the detailed discussion in this paper to two-dimensional potentials, so that the Poincaré section $\Sigma$ has one degree of freedom. In that case we may denote the eigensolutions of $\hat{h}$ by 


$$
\hat{h}|\tilde{k}\rangle=\left(k+\frac{1}{2}\right) \hbar|\tilde{k}\rangle,
$$

using a tilde to distinguish them from the chaotic eigenstates $|n\rangle$. Note then that the eigensolutions of $\hat{\mathcal{T}}$ are then

$$
\hat{\mathcal{T}}|\tilde{k}\rangle=e^{-(k+1 / 2) \alpha_{0}}|\tilde{k}\rangle=\frac{1}{\Lambda^{k+1 / 2}}|\tilde{k}\rangle,
$$

where $\Lambda=e^{\alpha_{0}}$. Notice that $\Lambda$ is the larger of the two eigenvalues $e^{ \pm \alpha_{0}}$ of $W$ (which are real even though $W$ is complex). Finally, we note that in double well problems for which the symmetry is an inversion $(x, y) \mapsto(-x,-y)$, the eigenvalues of $W$ as defined in [15] are negative and the eigensolutions of the tunnelling operator are of a similar form except that the eigenvalues are $\Lambda^{-k}|\Lambda|^{-1 / 2}$.

\section{B. Statistics using standard RMT}

A standard statistical model of eigenstates of chaotic systems is that their components in a generic basis are Gaussian-distributed. This assumption has been combined with various models of coupling to the continuum [3.10.11] to produce statistical distributions for the scaled tunnelling rates in the absence of scarring. The discussion in [11] forms the basis for our treatment of scarred distributions and we therefore summarise it here. We assume for ease of presentation that the system is time-reversal symmetric so the assumptions of the GOE are adopted. Results for GUE systems are derived similarly and are summarised at the end.

We expand the eigenstate $|n\rangle$ in an eigenbasis of $\hat{\mathcal{T}}$,

$$
|n\rangle=\sum_{k} x_{k}|\tilde{k}\rangle,
$$

suppressing the dependence of the coefficients $x_{k}$ on the chaotic-state index $n$. The normalisation of $|n\rangle$ is such that

$$
\left\langle\left|x_{k}\right|^{2}\right\rangle=1
$$

and in systems with time-reversal symmetry, the $x_{k}$ 's are real. The standard RMT model is that for large $N$, the $x_{k}$ 's are uncorrelated and distributed with a joint probability distribution

$$
P(\mathbf{x})=\prod_{k=0}^{N-1}\left[\frac{1}{\sqrt{2 \pi}} e^{-x_{k}^{2} / 2}\right]=\frac{1}{(2 \pi)^{N / 2}} e^{-\mathbf{x}^{T} \mathbf{x} / 2},
$$

where $\mathbf{x}=\left(x_{0}, x_{1}, x_{2}, \cdots\right)$. Given that (11) expresses each $y$ as a quadratic form

$$
y=\mathbf{x}^{T} T \mathbf{x}
$$

in $\mathbf{x}$, where $T$ is an $N \times N$ matrix representing $\hat{\mathcal{T}} / \operatorname{Tr} \hat{\mathcal{T}}$ in a $|\tilde{k}\rangle$-basis, we can write the distribution for $y$ in the form

$$
p(y)=\int \delta\left(y-\mathbf{x}^{T} T \mathbf{x}\right) P(\mathbf{x}) \mathrm{d} \mathbf{x} .
$$

Fourier-transforming with respect to $y$ gives the characteristic function, 


$$
\begin{aligned}
\tilde{p}(q) & =\int_{-\infty}^{\infty} e^{i q y} p(y) \mathrm{d} y \\
& =\frac{1}{(2 \pi)^{N / 2}} \int \exp \left[-\frac{1}{2} \mathbf{x}^{T}(I-2 i q T) \mathbf{x}\right] \mathrm{d} \mathbf{x} \\
& =\frac{1}{\sqrt{\operatorname{det}(I-2 i q T)}} .
\end{aligned}
$$

One finds similarly that

$$
\tilde{p}(q)=\frac{1}{\operatorname{det}(I-i q T)}
$$

in the absence of time-reversal symmetry.

These distributions have been shown in 11] to describe successfully the tunnelling rate statistics of chaotic double wells when the tunnelling route has a nonperiodic real extension. When the tunnelling route connects to a periodic orbit, however, strong deviations are found [11, 14] which we explain in the next sections using the idea of scarring.

\section{WAVEFUNCTION STATISTICS AND SCARRING}

It has been pointed out by Kaplan and coworkers that the Gaussian distribution in (2) does not describe wavefunction statistics if a basis state $|\tilde{k}\rangle$ is localised in phase space near a periodic orbit of the real classical dynamics (corresponding to the Poincaré map $F$ ). In tunnelling-rate statistics, this can lead to strong deviations from the distribution described the previous section if $\zeta_{0}$, in addition to being a fixed point of the complex map $\mathcal{F}$, is a fixed point or short periodic orbit of $F$. In this section we review elements of that argument and use them to calculate correlations among the components of $\mathbf{x}$. From this analysis there emerges a natural conjecture for generalising (2). The distributions we calculate on the basis of this conjecture are necessary to understand tunnelling but also, independently of the tunnelling problem, hold promise as a basis for understanding the statistics of scarring in a rather general way.

\section{A. A Gaussian hypothesis}

For a given ensemble of states with overlaps $\mathbf{x}=\left(x_{0}, x_{1}, x_{2}, \cdots\right)$, let us define a matrix of correlations $C$ whose elements are

$$
C_{l k}=\left\langle x_{l} x_{k}^{*}\right\rangle .
$$

The average is over the eigenstates $|n\rangle$ and will be made more precise below. We will in particular consider ensembles formed by the eigenstates $|n\rangle$ of $\hat{U}$ whose eigenangles lie in a subset of the unit circle. By varying the quantum dimension $N$, for example, we can consider ensembles corresponding to fixed classical maps $F$ and $\mathcal{F}$. If the joint probability distribution is of the form (2) or its GUE equivalent, we expect

$$
C=I,
$$

which says simply that $\left\langle\left|x_{k}\right|^{2}\right\rangle=1$ and that $x_{k}$ and $x_{l}$ are uncorrelated unless $k=l$. In order to avoid confusion with a correlation function defined below, we will henceforth refer to $C$ as the covariance matrix. We now show that deviations of $C$ from the identity can be calculated on the basis of linearised dynamics around the point $\zeta_{0}$. The construction begins with a calculation of quantum recurrence for basis states localised near $\zeta_{0}$ and is finished by a Fourier transformation which relates these to the averages $\left\langle x_{l} x_{k}^{*}\right\rangle$. 
Let $\{|\tilde{k}\rangle \mid k=0,1, \cdots\}$ be an eigenbasis of $\hat{\mathcal{T}}$ as defined in the previous section and suppose that $\zeta_{0}$ is a fixed point of the classical map $F$ corresponding to $\hat{U}$. We now argue that for some ensembles (7) cannot hold. Following [13] we define the correlation function

$$
\begin{aligned}
A_{l k}(t) & =\left\langle\tilde{l}\left|\hat{U}^{t}\right| \tilde{k}\right\rangle \\
& =\frac{1}{N} \sum_{n=1}^{N} x_{l} x_{k}^{*} e^{-i \theta_{n} t}
\end{aligned}
$$

and denote its Fourier transform by

$$
\begin{aligned}
S_{l k}(\theta) & =\sum_{t=-\infty}^{\infty} e^{i \theta t} A_{l k}(t) \\
& =\frac{2 \pi}{N} \sum_{n=1}^{N} x_{l} x_{k}^{*} \delta\left(\theta-\theta_{n}\right) .
\end{aligned}
$$

The correlation function is approximated semiclassically by a sum of the form

$$
A_{l k}(t) \approx \sum_{p} \mathcal{A}_{p} e^{2 \pi N i S_{p}}
$$

over classical orbits $p$, typically complex, which begin in and return to a small region around $\zeta_{0}$ after $t$ iterations of $F$, assuming $k$ and $l$ are $O(1)$. Each orbit contributes a term with an amplitude $\mathcal{A}_{p}$ which is independent of $N$ and an exponential $\exp \left[2 \pi N i S_{p}\right]$ with a rapidly-varying complex phase which decays exponentially with the distance of the contributing orbit from $\zeta_{0}$. The primary such orbit corresponds to the fixed point $\zeta_{0}$ itself. We are free to choose the phase of $\hat{U}$ so that the action of $\zeta_{0}$ vanishes (this amounts to a choice of the origin of the $\theta_{n}$ values). With that choice it is manifest that its contribution to $A_{l k}(t)$ is $N$-independent.

Consider averaging $A_{l k}(t)$ over a sequence of quantum systems with varying $N$ but identical classical limits. We find then that

$$
\left\langle A_{l k}(t)\right\rangle \approx \sum_{p} \mathcal{A}_{p}\left\langle e^{2 \pi N i S_{p}}\right\rangle
$$

and, with the exception of the fixed point $\zeta_{0}$ for which $S_{p}=0$, the contributions average to zero. The contribution from $\zeta_{0}$ can be obtained from linearised dynamics and we find that

$$
\left\langle A_{l k}(t)\right\rangle \approx A_{l k}^{\operatorname{lin}}(t) \equiv\left\langle\tilde{l}\left|\hat{U}_{\operatorname{lin}}^{t}\right| \tilde{k}\right\rangle
$$

where $\hat{U}_{\text {lin }}$ is the quantisation of a linearisation of the real map $F$ at $\zeta_{0}$. The linearised correlation function $A_{l k}^{\operatorname{lin}}(t)$ is calculated in the next section. It is $N$-independent and decays exponentially to zero with time when $k$ and $l$ are $O(1)$.

Performing the same averaging procedure on $S_{l k}(\theta)$ gives

$$
\left\langle S_{l k}(\theta)\right\rangle \approx S_{l k}^{\operatorname{lin}}(\theta) \equiv \sum_{t=-\infty}^{\infty} e^{-i \theta t} A_{l k}^{\operatorname{lin}}(t) .
$$

This is a real, $2 \pi$-periodic function of $\theta$ which is also $N$-independent. Suppose we form an ensemble of chaotic states by varying $N$ and selecting states for which $\theta_{n}$ lies within a narrow window centred about $\theta$. The eigenfunction expansion in (9) indicates that 


$$
C_{l k}(\theta)=\left\langle x_{l} x_{k}^{*}\right\rangle=\left\langle S_{l k}(\theta)\right\rangle \approx S_{l k}^{\operatorname{lin}}(\theta) .
$$

We find therefore that, if we produce an ensemble of overlaps from states within a narrow window of phase angle, the covariance matrix violates condition (7) and the joint probability distribution for $\mathbf{x}$ necessarily deviates from the standard RMT form.

The simplest distribution consistent with (11) is the Gaussian

$$
P(\mathbf{x} ; \theta)=\frac{1}{\left[(2 \pi / \beta)^{N} \operatorname{det} C(\theta)\right]^{\beta / 2}} e^{-\beta \mathbf{x}^{T} C^{-1}(\theta) \mathbf{x} / 2}
$$

where $\beta=1$ in the GOE case and $\beta=2$ in the case of GUE. We conjecture that these distributions govern the wavefunction statistics in the presence of scarring when states are taken from a small window of eigenphases. A full justification of this would require a detailed analysis of long orbits contributing to (10), along the lines of the nonlinear theory of scarring in [13]. We will not offer such an analysis here and assume (12) on the grounds of simplicity alone. This may be formalised by noting that (12) minimises the information content [17,18]

$$
\mathcal{I}[P(\mathbf{x})]=\int P(\mathbf{x}) \ln P(\mathbf{x}) \mathrm{d} \mathbf{x},
$$

subject to the constraint that the correlations are as written in (6) and that $N$ is large. Minimisation of information is not a proof, however, and ultimately we rely on detailed numerical testing to justify the choice of distribution. We now show in detail how $C(\theta)$ may be calculated and verify that the Gaussian hypothesis describes quite well the scarred wavefunction statistics of specific quantum-map models.

\section{B. Calculation of the covariance matrix}

Construction of the scarred distribution in (12) begins with the calculation of the linearised correlation function $A_{l k}^{\text {lin }}(t)$, from which the covariance matrix $C(\theta)$ is obtained by Fourier transformation. We show in this subsection how $A_{l k}^{\operatorname{lin}}(t)$ may be calculated from the monodromy matrices linearising real and complex dynamics around the fixed point $\zeta_{0}$ (corresponding to the maps $F$ and $\mathcal{F}$ respectively). In the main text we present enough detail that the method of calculation of $A_{l k}^{\operatorname{lin}}(t)$ should be clear and leave the derivation and other detailed discussion to appendices. Once $A_{l k}^{\operatorname{lin}}(t)$ has been constructed as outlined below, the covariance matrix is then easily calculated in practice using a FFT, for example.

It is shown in appendix A that $A_{l k}^{\text {lin }}(t)$ vanishes if $l$ and $k$ are not both even or both odd and may otherwise be calculated from the polar form

$$
A_{k+2 n, k}^{\operatorname{lin}}(t)=G_{k n}(\psi(t)) e^{i(k+1 / 2)(\phi(t)-\mu t \pi)+i n \phi(t)+i n \pi / 2},
$$

where the Maslov index $\mu$ and the angles $\psi(t)$ and $\phi(t)$ are defined by dynamics around $\zeta_{0}$ as described below. The amplitude is

$$
G_{k n}(\psi)=\sqrt{\frac{k !}{(k+2 n) !}} \frac{(2 n) !}{2^{n} n !} \sin ^{n} \psi \sqrt{\cos \psi} C_{k}^{n+1 / 2}(\cos \psi),
$$

where $C_{k}^{\alpha}(x)$ denotes a Gegenbauer polynomial. In the special case $n=0$ we get the autocorrelation function

$$
A_{k k}^{\operatorname{lin}}(t)=\sqrt{\cos \psi(t)} P_{k}(\cos \psi(t)) e^{i(k+1 / 2)(\phi(t)-\mu t \pi)},
$$

where $P_{k}(x)$ is a Legendre polynomial. We assume $n \geq 0$ and $t>0$ in (13) and use $A_{l k}^{\operatorname{lin} *}(-t)=A_{l k}^{\operatorname{lin}}(t)=$ $A_{k l}^{\operatorname{lin}}(t)$ to calculate the correlation function when $t<0$ or $l<k$. Note that this assumes a convention for 
the phases of the eigenstates $|\tilde{k}\rangle$ that is outlined at the end of appendix B. In particular, this convention leads to a covariance matrix $C(\theta)$ which is real-symmetric even in the case of GUE statistics; that is, while individual values of $x_{l} x_{k}^{*}$ are complex, the phase convention is such that the averages are real.

To define the angles $\phi(t)$ and $\psi(t)$, let $M_{0}$ be the real symplectic matrix linearising the unstable real dynamics around $\zeta_{0}$ and let

$$
W(z)=e^{i(\ln z) J K}
$$

be a generalisation of the complex symplectic matrix described in the the last section, reducing to that case when $z=e^{-\alpha_{0}}$. Euler expansion of the exponential in (14) leads to the identity

$$
\operatorname{Tr} W(z) M_{0}^{t}=(-1)^{\mu t}\left(m(t) z+\frac{m^{*}(t)}{z}\right),
$$

where

$$
m(t)=\cosh \rho t+i Q \sinh \rho t
$$

and where $(-1)^{\mu} e^{ \pm \rho}$ are the eigenvalues of $M_{0}$. The real parameter $Q$ follows the notation of 13] and is given by $Q=\cot \varphi$, where $\varphi$ is the angle between the stable and unstable manifolds of $M_{0}$. Note that the parameter $Q$ depends implicitly on $W$ because the angle $\varphi$ is calculated using the metric defined on phase space by $K$. Finally, we complete the explanation of (13) by defining the angles $\psi(t)$ and $\phi(t)$ implicitly using the polar decomposition

$$
m(t)=\sec \psi(t) e^{i \phi(t)}
$$

of $m(t)$. We may choose these angles to lie in the ranges $0<\psi(t)<\pi / 2$ and $-\psi(t)<\phi(t)<\psi(t)$ respectively (note that $\sec \psi \cos \phi=\cosh \rho t>1$ ) and, with this restriction on $\phi(t)$, the half-angle in (13) is well-defined.

Note that, as $t \rightarrow \infty$, we find

$$
\cos \psi(t) \sim \frac{2}{\sqrt{1+Q^{2}}} e^{-\rho t}
$$

and the correlation function in (13) therefore decays at the exponential rate $e^{-\rho t / 2}$ when $k$ is even and at the rate $e^{-3 \rho t / 2}$ when $k$ is odd (since $C_{k}^{n+1 / 2}$ is then an odd polynomial). Less obvious from (13) is the fact that the correlation function also decays for fixed $t$ with increasing $k$ and $l=k+2 n$. This is a reflection of the fact that more excited states of the oscillator $\hat{h}$ are less localised at the fixed point $\zeta_{0}$ and therefore less affected by scarring.

When the linear correlation function is Fourier-transformed, we therefore get a covariance matrix $C_{l k}(\theta)$ which can be decomposed into two blocks corresponding to odd and even $l$ and $k$ and whose elements approach those of the identity matrix $\left(C_{l k}(\theta) \rightarrow \delta_{l k}\right)$ as $l$ and $k$ increase. We expect scarring therefore to affect the statistics of $x_{k}$ significantly for relatively small values of $k$ and to have little effect when $k$ is large. This exponential convergence towards identity means that the matrix $C-I$ is trace-class and the determinant of $C$ exists in the limit $N \rightarrow \infty$. In the next subsection we calculate the resulting covariance matrix for some model quantum maps and show that the Gaussian hypothesis provides a good model for the wavefunction statistics of scarred systems.

\section{Testing the Gaussian hypothesis}

We test the Gaussian hypothesis using a model system in which $\hat{U}$ quantises the perturbed cat map, 


$$
F_{\epsilon}=F_{0} \circ M_{\epsilon}
$$

where

$$
F_{0}:\left(\begin{array}{c}
q \\
p
\end{array}\right) \mapsto\left(\begin{array}{cc}
1 & 1 \\
1 & 2
\end{array}\right)\left(\begin{array}{c}
q \\
p
\end{array}\right) \quad \bmod 1
$$

is the standard cat map and

$$
M_{\epsilon}:\left(\begin{array}{c}
q \\
p
\end{array}\right) \mapsto\left(\begin{array}{c}
q-\epsilon \sin 2 \pi p \\
p
\end{array}\right) \quad \bmod 1
$$

is a kicked Harper map. For sufficiently small values of the parameter $\epsilon$ this map shares the hyperbolic structure of the unperturbed map but not its nongeneric degeneracies. Details of the quantisation of these maps for Hilbert spaces of arbitrary dimension $N$ may be found in [19,20].

The map $F_{\epsilon}$ has a symmetry of inversion about the origin, which is always therefore a fixed point. It also has a less obvious time-reversal symmetry. One finds that

$$
P F_{\epsilon} P=F_{\epsilon}^{-1},
$$

where

$$
P:\left(\begin{array}{c}
q \\
p
\end{array}\right) \mapsto\left(\begin{array}{ll}
-1 & 0 \\
-1 & 1
\end{array}\right)\left(\begin{array}{l}
q \\
p
\end{array}\right) \quad \bmod 1 .
$$

Note that $P$ is antisymplectic and that $P^{2}=I$. The map therefore allows us to test statistics in the GOE scenario. (A systematic discussion of such time-reversing symmetries can be found in [21]).

To complete our model of a tunnelling system, we construct an analog of the tunnelling operator. Consider the Harper-like Hamiltonian

$$
h(q, p)=2-\cos \pi(2 p-q)-\cos 2 \pi q .
$$

This is invariant under the time-reversal symmetry $P$ and has a minimum at the origin in phase space. The function does not have the full periodicity of the torus in $q$ but, by placing the resulting discontinuity at $q=1 / 2$, may be quantised in such a way that the ground and first few excited states of its quantisation $\hat{h}$ are localised near the origin. These are then approximated semiclassically by those of the harmonic oscillator $(\hat{p}-\hat{q} / 2)^{2}+\hat{q}^{2}$. The statistics of the components $x_{k}$ of chaotic eigenstates of $\hat{U}$ in an eigenbasis of $\hat{h}$ therefore provide a test of the Gaussian hypothesis outlined in the previous subsection and since $\hat{U}$ and $\hat{h}$ share a time-reversal symmetry, the GOE hypothesis is appropriate.
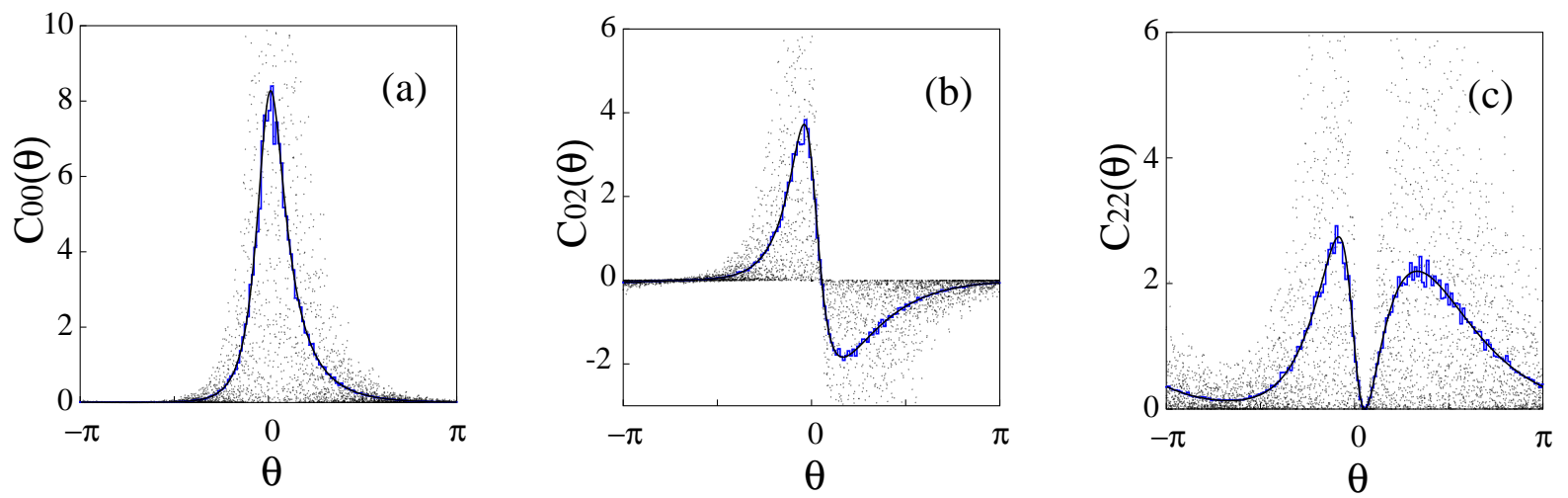

FIG. 1. The smooth curves are calculated using our prediction for the covariance matrix as a function of phase angle $\theta$. We show $C_{00}(\theta), C_{02}(\theta)$ and $C_{22}(\theta)$ in $(a),(b)$ and $(c)$ respectively. In each case we find good agreement with the step curve, which represents the local average obtained by binning components of the eigenstates of $\hat{U}$ for $\epsilon=0.1$ and $N$ between 100 and 700 . The points represent overlaps of individual states. 
We first consider the prediction for the covariance matrix $C(\theta)$. The element $C_{00}(\theta)$, which corresponds to the overlap statistics of a Gaussian wavepacket (defined as the ground state of $\hat{h}$ ), has been considered extensively by Kaplan and coworkers. For comparison with later calculation it is plotted as a function of $\theta$ in Fig. 11 $(a)$ for the map $F_{\epsilon}$ with $\epsilon=0.1$. Following [13], we refer to this curve as the spectral envelope. We expect larger than average overlaps where $C_{00}(\theta)>1$ and we will refer to this as the scarred region. The rest of the interval is the antiscarred region, where we expect smaller than average overlaps. The points in the figure give a representative sample of individual overlaps. There is also a curve representing a binned average of these overlaps which is obtained by accumulating quantum data for even values of $N$ between 100 and 700. It is also important for our analysis to understand the statistics of $x_{l} x_{k}$ when $l$ and $k$ lable excited states of $\hat{h}$. We therefore make an analogous comparison with quantum data for $C_{02}(\theta)$ and $C_{22}(\theta)$ in Figs. 1 $1(b)$ and $1(c)$ respectively. Note the different vertical scales in these cases. In all cases the average obtained by binning quantum data agrees well with the prediction $C_{l k}(\theta)$ obtained by Fourier-transforming the correlation function. This is to be expected since we have a relatively direct argument showing that this should be so. Less obvious is that the distribution of individual overlaps about these averages should be governed by the Gaussian hypothesis, since we do not have a proof for that. That this assumption can be verified numerically (below) is therefore nontrivial.

To compare the distributions about the mean $C_{l k}(\theta)$, lets us denote

$$
\xi_{l k}=\operatorname{Re} x_{l} x_{k}^{*}=\mathbf{x}^{\dagger} B_{l k} \mathbf{x},
$$

where $B_{l k}$ is a symmetric matrix whose $i j^{\text {th }}$ entry is $\left(\delta_{i l} \delta_{j k}+\delta_{i k} \delta_{j l}\right) / 2$. (For the moment we allow either GUE or GOE statistics even though all the numerical calculations we present are for the GOE case). Similarly to the calculation of $p(y)$ in section III, we find that the local distribution $p\left(\xi_{l k} ; \theta\right)$ is predicted by the Gaussian hypothesis to have the Fourier transform

$$
\begin{aligned}
\tilde{p}_{l k}(q ; \theta) & =\int_{-\infty}^{\infty} e^{i q \xi_{l k}} p\left(\xi_{l k} ; \theta\right) \mathrm{d} \xi_{l k} \\
& =\frac{1}{\left[(2 \pi / \beta)^{N} \operatorname{det} C(\theta)\right]^{\beta / 2}} \int \exp \left[-\frac{1}{2} \mathbf{x}^{\dagger}\left(C^{-1}(\theta)-\frac{2 i q}{\beta} B_{l k}\right) \mathbf{x}\right] \mathrm{d} \mathbf{x} \\
& =\left[\operatorname{det}\left(I-\frac{2 i q}{\beta} B_{l k} C(\theta)\right)\right]^{-\beta / 2} .
\end{aligned}
$$

The determinant simplifies on expansion to

$$
\operatorname{det}\left(I-\frac{2 i q}{\beta} B_{l k} C(\theta)\right)=1-\frac{2 i q}{\beta} C_{l k}+\frac{q^{2}}{\beta^{2}}\left(C_{l l} C_{k k}-C_{l k}^{2}\right)
$$

and Fourier transformation of (15) then gives

$$
p\left(\xi_{l k} ; \theta\right)=e^{\beta \xi_{l k} C_{l k} / D_{l k}} f\left(\xi_{l k} ; D_{l k}, B_{l k}\right) .
$$

Here, $D_{l k}=C_{l l} C_{k k}-C_{l k}^{2}, B_{l k}=\sqrt{C_{l l} C_{k k}}$ and $f(\xi ; D, B)$ is an even function of $\xi$, defined by

$$
f(\xi ; D, B)= \begin{cases}\frac{1}{\pi D^{1 / 2}} K_{0}(B \xi / D), & \text { for GOE and } \\ \frac{1}{B} \exp [-2 B|\xi| / D], & \text { for GUE }\end{cases}
$$

(in which $K_{0}(z)$ is a modified Bessel function). 

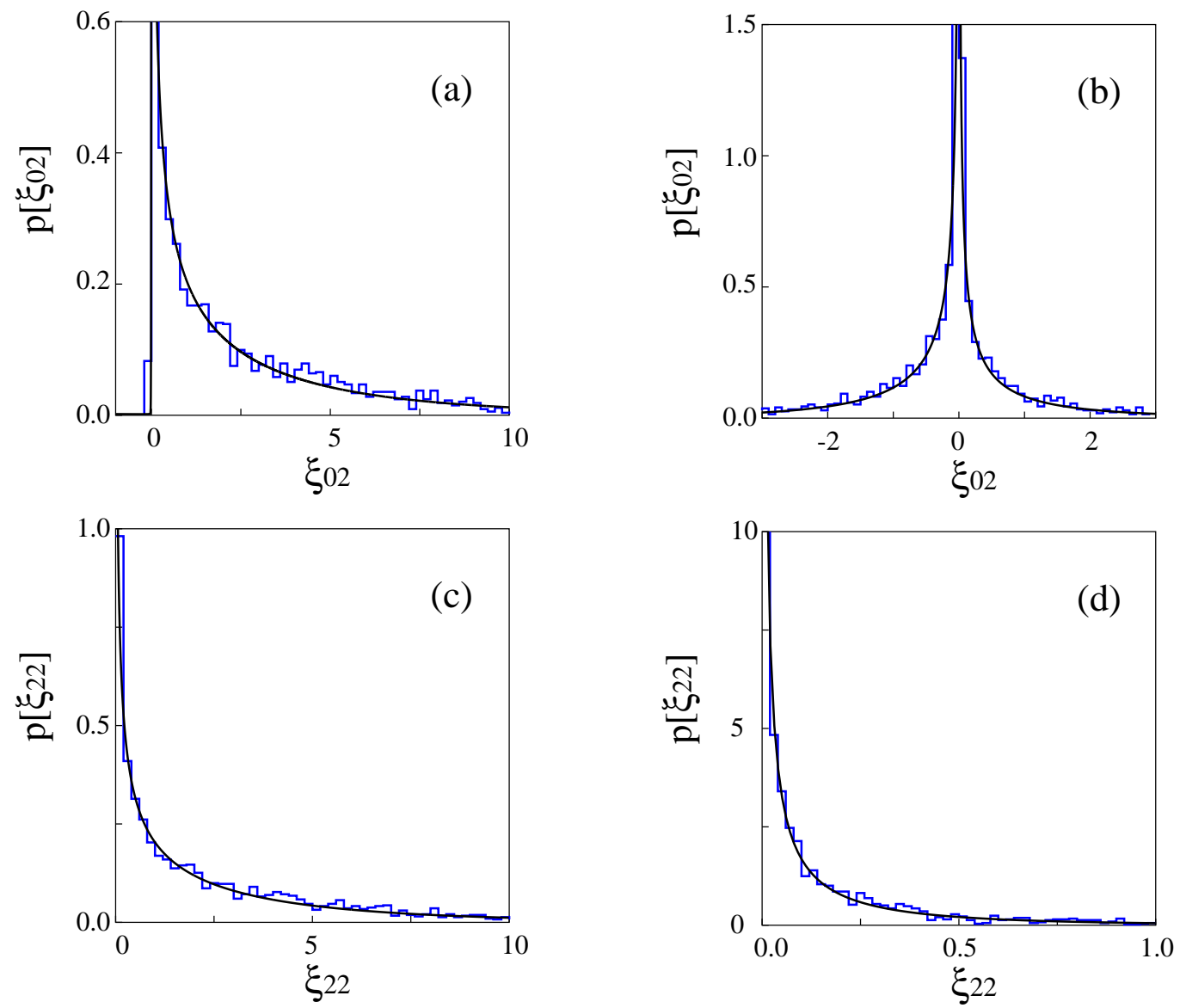

FIG. 2. In (a) and (b) the statistical distribution of $\xi_{02}=x_{0} x_{2}$ over the intervals $\mathcal{I}=(-0.14 \pi,-0.06 \pi)$ and $\mathcal{I}=(0.02 \pi, 0.10 \pi)$ respectively are compared with the prediction of the Gaussian hypothesis. The two intervals chosen correspond respectively to regions of phase angle which are scarred and (relatively) antiscarred. Figures (c) and (d) show the statistics of $\xi_{22}=x_{2}^{2}$ in the same scarred and antiscarred intervals. Note the different scales in the scarred and antiscarred cases.

When $l=k$ we find in the GOE case that

$$
p\left(\xi_{k k} ; \theta\right)=\frac{\xi_{k k}^{-1 / 2}}{\sqrt{2 \pi C_{k k}(\theta)}} e^{-\xi_{k k} / 2 C_{k k}(\theta)},
$$

which is just the Porter-Thomas distribution scaled so that $\left\langle\xi_{k k}\right\rangle=C_{k k}(\theta)$, consistent with [13].

In order to test these predictions with reasonable statistics, we create ensembles by selecting states with phase angles restricted to subsets $\mathcal{I}$ of the unit circle and compare their statistics with the accumulated distribution

$$
p_{\mathcal{I}}\left(\xi_{l k}\right)=\frac{1}{\mu(\mathcal{I})} \int_{\mathcal{I}} p\left(\xi_{l k} ; \theta\right) \mathrm{d} \theta
$$

where $\mu(\mathcal{I})=\int_{\mathcal{I}} \mathrm{d} \theta$. These ensembles provide averages that are local in $\theta$ while allowing sufficient data to make meaningful numerical tests. The resulting statistical distributions are compared in Fig. 2 with the 
statistics of $\xi_{02}=x_{0} x_{2}$ and $\xi_{22}=x_{2}^{2}$. In each case the statistics in two subintervals are shown: one covering a region where there is scarring (larger than average overlaps) and one in a region where there is antiscarring (smaller than average overlaps). In all cases the Gaussian hypothesis works well in describing the whole distribution. Note that similar agreement has been found for models with GUE statistics (which are not shown here in the interests of brevity).
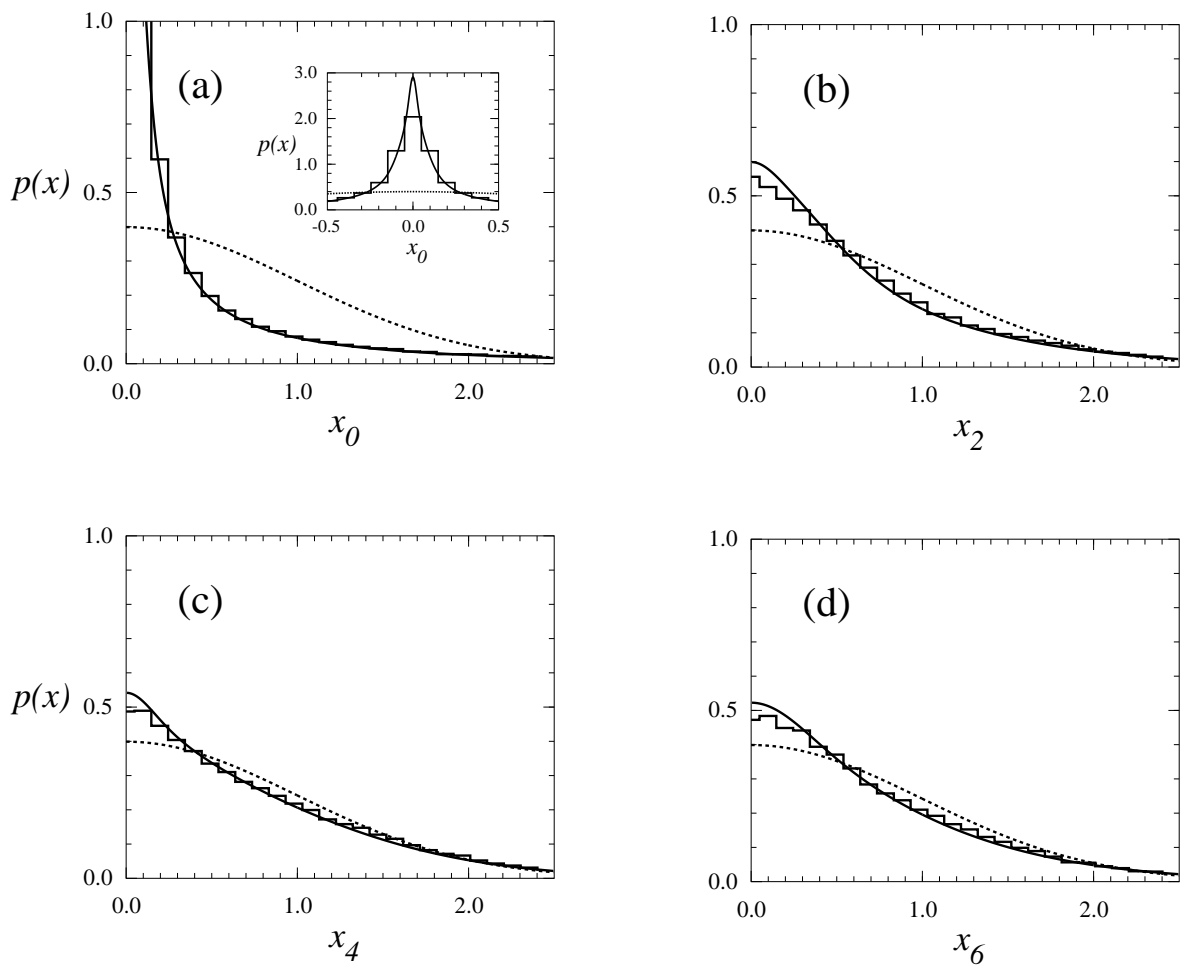

FIG. 3. The distributions of $x_{k}$, accumulated over the whole unit circle of phase angle, are shown in (a)-(d) for $k=0,2,4,6$ respectively. The insert in (a) shows the tip of the distribution. The Gaussian hypothesis (solid curve) is in good agreement with the quantum data (histogram) over the whole distribution. In each figure the dashed curve is the normal, unscarred, RMT prediction. The scarred distributions approach this RMT case as $k$ increases (although not necessarily monotonically in general).

We also show, in Fig. 3, distributions for the individual overlaps $x_{k}$. The local distributions $p\left(x_{k} ; \theta\right)$ are Gaussians scaled to that the variances are $C_{k k}(\theta)$, analogously to (3). We then define accumulated distributions by integrating over an interval $\mathcal{I}$ with respect to $\theta$, as in (17). In practice, the most natural ensemble is formed by averaging over the whole unit circle, corresponding to taking $\mathcal{I}=[0,2 \pi)$. A sequence of such distributions is shown in Fig. 3 corresponding to $k=0,2,4,6$. One sees that the distribution approaches the normal GOE prediction as $k$ increases but that for smaller values of $k$ the deviation from GOE is quite strong. Note that the Gaussian hypothesis describes quite accurately the statistics for moderate values of $x_{k}$ as well as in the tails which correspond to the strong scarring limit. There is some underestimation in the antiscarring limit where $x_{k}$ is small, but the overall agreement is good over the whole range of values of $x_{k}$. Similar or better agreement has been found in all the models we have examined.

All the numerical evidence we have collected indicates strongly that the Gaussian hypothesis accurately describes the complete statistics of chaotic eigenstates in a neighbourhood of a periodic orbit. While mo- 
tivation for extending the linear theory of scarring to a complete basis has initially come from a desire to treat tunnelling, we note that the results in this section are interesting from the point of view of scarring alone. The covariance matrix $C(\theta)$ provides a means of characterising the eigenstate statistics in the neighbourhood of a periodic orbit in a way that is independent of the choice of test state. We can accomodate the characterisation of statistics using a basis other than $\{|\tilde{k}\rangle\}$ simply by altering the covariance matrix using a similarity transformation. Ideally, any basis we use should in the interests of compactness cover the immediate neighbourhood of the periodic orbit (as measured by Wigner functions, for example) with as few members as possible, but we are otherwise then free to manipulate those basis elements at will. In addition to being essentially basis-independent in this way, such a characterisation is complete, in the sense if we choose a subset of the basis which covers a semiclassical neighbourhood of the periodic orbit, the statistics of any other measure of the eigenstate near the periodic orbit could in principle then be determined from the corresponding sub-block of the covariance matrix.

Note finally that the deviations from RMT of the statistics of low-lying components of the eigenstate persist, and in fact remain fixed, in the semiclassical limit. This can be understood intuitively to derive from the fact that as $\hbar \rightarrow 0$ (or $N \rightarrow \infty$ ) the support of a harmonic oscillator basis state shrinks at the same rate as the semiclassical footprint of the periodic orbit. In $\Sigma$, the area occupied by each decays as $O(\hbar)$ in the semiclassical limit. This semiclassical shrinking of the test states we use allows the scarring effect to remain fixed while other measures of scarring (such as the value of a wavefunction at a fixed point in space) lead to a vanishing effect in the semiclassical limit.

\section{TUNNELLING STATISTICS WITH SCARRING}

The scarred wavefunction statistics described in the previous section are now used to derive distributions for the anomalous tunnelling rate statistics of [11,14]. The first step is to outline how the discussion for maps in the preceding section may be adapted to the statistics of states in potential-well problems and then we can describe how these ideas are used to construct distributions for tunnelling rates.

While the formalism of tunnelling is presented in 11,15] in terms of quantum maps and their eigenstates, quantisation of a potential-well problem leads more naturally to wavefunctions in a full Hilbert space and at first sight it is not obvious how to relate the two descriptions. Faced with a numerically-produced wavefunction, for example, there is no exact, purely quantum-mechanical way of defining a corresponding state in a finite-dimensional Hilbert space; all existing descriptions are semiclassical in nature. Our task is made easier by the fact that tunnelling depends only on properties of the state in a small region of phase space (or of a Poincaré section in the reduced description) surrounding a tunnelling orbit. Within that region, the dynamical properties of the tunnelling orbit have been used in [15] to give an explicit prescription for the definition of a Wigner function on a Poincaré section. We will not repeat the details of that prescription here but state simply that a recipe exists for turning an eigenfunction $\psi_{n}(x, y)$ of a Hamiltonian with potential $V(x, y)$ into a Wigner function $\mathcal{W}_{n}\left(y, p_{y}\right)$ on a Poincaré section $\Sigma$ defined by $x=x_{0}$ and on which $\left(y, p_{y}\right)$ are canonical coordinates. This Wigner function is invariant near the tunnelling orbit under iteration of a semiclassical quantisation of the Poincaré map [16]. With appropriate normalisation this construction allows us to compute overlaps, using

$$
|\langle n \mid \tilde{k}\rangle|^{2}=\int_{\Sigma} \mathrm{d} y \mathrm{~d} p_{y} \mathcal{W}_{n}\left(y, p_{y}\right) \tilde{\mathcal{W}}_{k}\left(y, p_{y}\right)
$$

between a reduced chaotic state $|n\rangle$ and an eigenstate $|\tilde{k}\rangle$ of a harmonic oscillator Hamiltonian $h\left(y, p_{y}\right)$ defined on the Poincaré section $\Sigma$.

We now check that these overlaps are described statistically by the distributions derived in the preceding section. Until now the discussion has been given entirely in terms of simple quantum maps for which the chaotic spectrum is described in terms of eigenangles on the unit circle. In physical problems the spectrum 
is more naturally described in terms of energy levels on the real line and we must connect the two pictures before proceeding. A detailed connection can be made using the transfer-operator approach of [16], but for present purposes it is sufficient to identify the part of the spectrum corresponding to a narrow segment of the spectral envelope (so that we may partition states into ensembles with simple Gaussian overlap statistics). A continuous-time analog of the phase angle $\theta$ is provided by the action $S(E)$ of the periodic orbit responsible for scarring [13]. We therefore partition the quantum states into ensembles for which $\theta=S(E) / \hbar$ lies in narrow intervals, mod $2 \pi$. We assert that the statistics of the overlaps in (18) for each such ensemble should be a nonisotropic Gaussian such as described in (12).
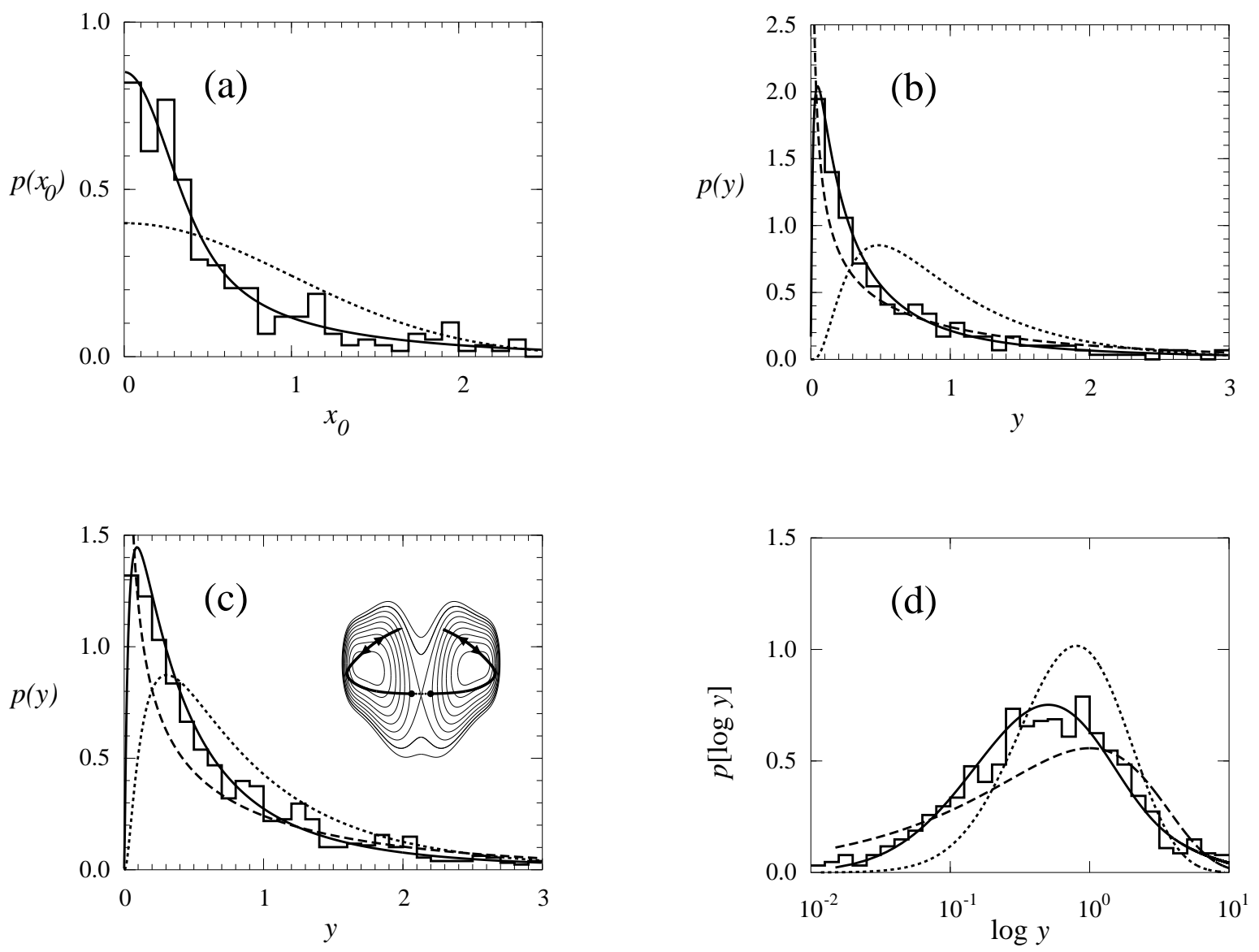

FIG. 4. In (a) the theoretical distribution (solid curve) for overlaps with a harmonic-oscillator ground state is compared with quantum data (histogram) for the $(y$-symmetric) potential with $(\mu, \nu, \sigma)=(0.1,0,0)$. The energy is fixed at the value $E=0.9$ (where the potential saddle has energy $E=1$ ) and $\hbar$ is quantised in the quantum calculation. The histogram represents 392 states with $48<1 / \hbar<75.77$, consisting of 12 periods of the spectral envelop function. In $(b)$ we show the corresponding distribution of rescaled tunnelling rates. In addition to the standard RMT prediction without scarring (short-dashed curve) we also show the Porter-Thomas distribution as a long-dashed curve. In $(c)$ and $(d)$ we present tunnelling rate statistics, using linear and log scales respectively, for the potential with $(\mu, \nu, \sigma)=(0.25,0.40,0.254)$. In this case states 1480 states with $40<1 / \hbar<106.47$, representing 54 periods of the spectral envelop function, are used. This potential is not symmetric in $y$ but the real extension of the tunnelling orbit is still periodic (inset). As in $(a)$ and $(b)$, the short-dashed curve represents a RMT calculation without scarring, the solid curve the scarring distribution and the long-dashed curve shows the Porter-Thomas distribution. 
We test this assertion using the two-dimensional double-well potential

$$
V(x, y)=\left(x^{2}-1\right)^{4}+x^{2} y^{2}+\mu y^{2}+\nu y+\sigma x^{2} y
$$

whose dynamics are predominantly chaotic near the saddle energy for appropriate parameter values and which we will later use to test tunnelling rate statistics. In practice we form statistical ensembles by fixing the energy and quantising $\hbar$ and in this way classical-dynamical parameters are held fixed throughout the spectrum. Our system does not allow the accumulation of sufficient data to test the distributions meaningfully for a fixed value of $\theta=S(E) / \hbar$ and we must instead pass straight to a distribution in which all states are combined, of the kind illustrated in Fig. 3. We show in Fig. 因 $(a)$ the accumulated distribution $p\left(x_{0}\right)$ of overlaps with a harmonic oscillator ground state (with $k=0$ ) for the parameter values $(\mu, \nu, \sigma)=(0.1,0,0)$. The potential in this case has a symmetry of reflection in $y$ and therefore has a real periodic orbit connecting to a tunnelling orbit in the $x$-axis. The harmonic oscillator used is the one which generates the monodromy matrix $W$ of the tunnelling orbit and from which the tunnelling operator is constructed. The distribution derived from the Gaussian hypothesis describes the quantum data well and indicates that it is a reasonable basis from which to tackle tunnelling-rate statistics.

To describe tunnelling-rate statistics, it is convenient to begin once again with an ensemble taken from a fixed part of the spectral envelope (with $\theta=S(E) / \hbar$ fixed $\bmod 2 \pi$ ). Repeating the derivation of (15), with $B_{l k}$ relaced by the matrix $T$ representing the scaled tunnelling operator, we obtain

$$
p(y ; \theta)=\frac{1}{2 \pi} \int_{-\infty}^{\infty} e^{-i q y} \tilde{p}(q, \theta) \mathrm{d} q,
$$

where

$$
\tilde{p}(q, \theta)=\left[\operatorname{det}\left(I-\frac{2 i q}{\beta} T C(\theta)\right)\right]^{-\beta / 2} .
$$

Our numerical calculations are made for GOE systems, in which case $\beta=1$. (Note that while the mechanics of the calculation are similar to those leading to (15), the quantities under investigation are very different in each case.) This distribution describes an ensemble of tunnelling rates taken with a fixed value of $\theta$. As was the case for overlap statistics, it is not possible with our present model to produce enough data to test the distribution for narrow ranges of $\theta$ and instead we make a comparison in which states from all parts of the spectral envelope are combined. Theoretically, we produce a combined distribution describing such collective statistics by averaging $p(y, \theta)$ over $0 \leq \theta<2 \pi$.

Comparison of the resulting distribution with quantum data for the potential $V(x, y)$, with the same parameter values as in Fig. 1( $(a)$, is shown in Fig. $1(b)$ as a solid curve. Agreement with the scarred distribution is good and there is marked deviation from the prediction of standard RMT (short-dashed curve). Also shown for comparison is the Porter-Thomas distribution [14] (long-dashed curve).

Systems with symmetry such as that used for Figs. $4(a)$ and $4(b)$ are expected generically to show scarred statistics of the kind described in this paper. Nonsymmetric systems may also have scarred statistics, however, if parameters are tuned so that the real extension of the tunnelling orbit is periodic. We show tunnelling rate statistics in Figs. $1(c)$ and $1(d)$ for one such potential, corresponding to the parameter values $(\mu, \nu, \sigma)=(0.25,0.40,0.254)$. Quantum data for this potential were used in 11] to illustrate that dynamics could affect tunnelling statistics and the present theory gives a quantitative explanation of the qualitative observations made there. In Fig. 田(c) we compare the scarred $p(y)$ (solid curve) with the standard RMT prediction (short-dashed curve) and the Porter-Thomas distribution (long-dashed curve).

Note that while the scarred distribution appears not to differ strongly from the Porter-Thomas distribution

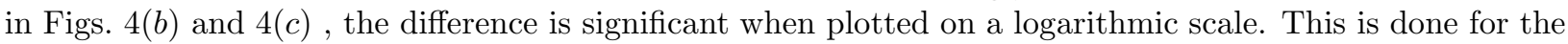
nonsymmetric potential in Fig. $1(d)$ (and omitted for the symmetric potential in the interests of space). We also note that in the case of the symmetric potential the first hundred or so states (omitted from Figs. E $(a)$ 
and $⿴(b))$ give a distribution that seems better described by Porter-Thomas, though the scarred distribution unambiguously describes better the more highly excited states corresponding. This semiclassical limit seems to be approached more rapidly for the nonsymetric potential.

We note that while the detailed discussion has been presented here for two-dimensional systems, the general theory will also work in higher dimensions. In particular, for example, (19) holds except that the matrices $T$ and $C(\theta)$ have additional structure. Generalisation of $T$ to higher dimensions is straighforward (see [15]) and, for $C(\theta)$, the generation of the autocorrelation function as described in the appendices may also be extended, though there are now more than the three classical parameters $\left(\operatorname{Tr} W, \operatorname{Tr} M_{0}, Q\right)$, controlling quantum recurrence.

The success of scarring theory in describing the tunnelling-rate distribution therefore confirms the hypothesis of 11] that dynamical details of such low-dimensional systems can dominate the statistics of tunnelling and be responsible for strong deviation from RMT. The deviations we have seen are expected to be common in systems with discrete symmetry, such as the double well potential we have treated in Figs. $1(a)$ and Q $(b)$. Deviation will also be seen in more generic systems, however, as parameters are tuned to make the real extension of the tunnelling orbit periodic [11. Such sensitive dependence of experimentally-accessible data on system parameters and dynamics might be useful, for example, as a means of probing the internal dynamics of relatively complex systems.

\section{CONCLUSION}

We have shown that wavefunction statistics in the neighbourhood of a fixed point or short periodic orbit are well described by a normal distribution which allows for correlations between wavefunction components along different basis elements. We have also shown that this wavefunction distribution can be used to describe scarring anomalies previously detected in tunnelling-rate statistics.

Scarring effects lead to strong deviations from the standard distributions of RMT in the neighbourhood of short periodic orbits. To characterise these deviations fully, we examined the components of the eigenstates of a chaotic map in the eigenbasis of a harmonic oscillator centred on a periodic orbit. The low-lying states of such an eigenbasis are localised around the corresponding periodic orbit and overlaps with a chaotic wavefunction are strongly affected by scarring. In appropriate subintervals of the chaotic spectrum, we have proposed that the joint probability distribution describing the corresponding components of chaotic eigenstates remain normal as in standard RMT but has a nontrivial covariance matrix (whose elements are completely determined by linearised dynamics around the periodic orbit). This hypothesis suffices to describe accurately all the measures of deviation from RMT that we have examined. The collective statistics of chaotic wavefunctions taken from a complete chaotic spectrum are described by superimposing these distributions. Deviations from RMT remain strong (and are effectively reproduced by the theory) in these combined ensembles, which are more natural to work with from a practical point of view.

We believe that examining the wavefunction statistics in a complete basis such as this provides a promising technique for understanding scarring in general terms. The description is essentially basis-independent and also completely determines the nature of wavefunction statistics near a periodic orbit.

The assumption of normal deviation from RMT also allows the effect of scarring to be incorporated very naturally into the calculation of tunnelling rate statistics. The theory was tested on a two-dimensional double-well potential with chaotic dynamics and found to describe the statistics of its eigenfcuntions and energy-level splittings well. Scarring can account for quite strong deviation from standard RMT in this system. In this way we might use tunnelling and the statistics of tunnelling to probe sensitively the internal dynamics of unstable systems. 


\section{Acknowledgements}

SC Creagh and SY Lee are supported by the EPSRC under the Fast Stream scheme. SY Lee also acknowledges support from KOSEF.

[1] C. E. Porter and R. G. Thomas, Phys. Rev. 104, 483 (1956).

[2] W. F. Polik, D. R. Guyer and C. B. Moore, J. Chem. Phys. 92, 3453 (1990).

[3] W. H. Miller, R. Hernandez and C. B. Moore, J. Chem. Phys. 93, 5657 (1990).

[4] A. C. Gentile, S. A. Schofield and P. G. Wolynes, J. Chem. Phys. 98, 7898 (1993).

[5] M. R. Wedlock R. Jost and T. R. Rizzo, J. Chem. Phys. 107, 10344 (1997).

[6] J. Hauschildt et al, Chem. Phys. Lett. 300, 569 (1999).

[7] A. Callegari et al, J. Chem. Phys. 111, 7359 (1999).

[8] H. Nakamura and S. Kato, J. Chem. Phys. 112, 1785 (2000).

[9] R. A. Jalabert, A. D. Stone and Y. Alhassid, Phys. Rev. Lett. 68, 3468 (1992); A. M. Chang et al., Phys. Rev. Lett. 761695 (1996); J. A. Folk et al., Phys. Rev. Lett. 761699 (1996).

[10] Y. Alhassid and C. H. Lewenkopf, Phys. Rev. Lett. 75, 3922 (1995); Phys. Rev. B 55, 7749 (1997); Y. Alhassid, J. N. Hormuzdiar and N. D. Whelan, Phys. Rev. B 58, 4866 (1998); E. E. Narimanov et al., Phys. Rev. Lett. 83, 2640 (1999).

[11] S. C. Creagh and N. D. Whelan, Phys. Rev. Lett. 84, 4084 (2000).

[12] S. W. McDonald, PhD Thesis, Lawrence Berkeley Laboratory LBL 14873 (1983). E. J. Heller, in Quantum Chaos and Statistical Nuclear Physics, T. H. Seligmann and H. Nishioka (eds.), Lecture notes in Physics 263, (Springer-Verlag, Berlin, 1986).

[13] L. Kaplan, Phys. Rev. Lett. 80, 2582 (1998); L. Kaplan and E. J. Heller, Ann. Phys. 264, 171 (1998).

[14] W. E. Bies, L. Kaplan and E. J. Heller, to appear in Phys. Rev. E.

[15] S. C. Creagh and N. D. Whelan, Ann. Phys. 272, 196 (1999).

[16] E. B. Bogomolny, Nonlinearity 5, 805 (1992).

[17] R. Balian, Nuovo Cimento B 57, 183 (1968).

[18] M. L. Mehta Random Matrices, Academic Press (1991).

[19] M. B. de Matos and A. M. de Almeida, Ann. Phys. 235, 1, (1994).

[20] S. C. Creagh, CHAOS 5, 477 (1995).

[21] J. P. Keating and F. Mezzadri, Nonlinearity 13, 747 (2000).

[22] M. Tabor, Chaos and Integrability in Nonlinear Dynamics (Wiley, 1989).

\section{APPENDIX A: GENERATING THE CORRELATION FUNCTION}

We calculate the correlation function $A_{l k}(t)$ defined in section III using a generating function

$$
G(w, z, t)=\operatorname{Tr} e^{w \hat{a}} \hat{\mathcal{T}}(z) \hat{U}_{\text {lin }}^{t},
$$

where $\hat{a}$ is an annihilation operator for the harmonic oscillator $\hat{h}$ and

$$
\hat{\mathcal{T}}(z)=e^{(\ln z) \hat{h} / \hbar}
$$

reduces to the tunnelling operator when $z=e^{-\alpha_{0}}$. Each of the three operators in this sequence is the quantisation of either a linear symplectic transformation or a phase-space translation, possibly complex. The unitary operator $\hat{U}_{\text {lin }}^{t}$ quantises a real symplectic matrix $M_{0}^{t}$ which linearises the real unstable motion near 
$\zeta_{0}$. The operator $\hat{\mathcal{T}}(z)$, which is nonunitary, quantises the complex symplectic matrix $W(z)$ defined in (14). Finally, the operator $e^{w \hat{a}}$ can be interpreted as a translation along the complex phase-space displacement

$$
\delta \zeta=-w \sqrt{\frac{\hbar}{2}}\left(\begin{array}{l}
1 \\
i
\end{array}\right) .
$$

The combined operator therefore quantises the affine transformation

$$
\zeta \mapsto W(z) M_{0}^{t} \zeta+\delta \zeta .
$$

Because the transformation is affine, the trace formula may be used to evaluate the right hand side (A1) exactly. It is shown in appendix B that this gives

$$
G(w, z, t)=\frac{1}{\sqrt{\Delta(z, t)}} \exp \left[(-1)^{\mu t} \frac{i w^{2}}{2} \frac{z}{\Delta(z, t)} \tan \psi(t)\right]
$$

where

$$
\Delta(z, t)=\operatorname{Tr} W(z) M_{0}^{t}-2=(-1)^{\mu t}\left(m(t) z+\frac{m^{*}(t)}{z}\right)-2
$$

and $m(t)=e^{i \phi(t)} \sec \psi(t)$ as described in Sec. III. Note that $G(w, z, t)$ is independent of $\hbar$. Note also that since $\Delta(z, t)$ is complex, care must be taken in deciding the branch of the square root in (A3) - the branch is chosen using the Maslov index (computed from real dynamics), so that $\Delta(z, t)$ reduces to the standard formula for real maps when $z \rightarrow 1$.

Expanding the right hand side of $(\mathrm{A} 3 \mathrm{n})$ gives

$$
G(w, z, t)=\sum_{n=0}^{\infty}(-1)^{n \mu t} \frac{i^{n} w^{2 n}}{2^{n} n !} \sum_{k=0}^{\infty} z^{k+2 n+1 / 2} \sin ^{n} \psi \sqrt{\cos \psi} C_{k}^{n+1 / 2}(\cos \psi) e^{i(n+k+1 / 2)(\phi-\mu t \pi)},
$$

where $C_{k}^{n+1 / 2}(\cos \psi)$ are Gegenbauer polynomials defined by the generating function

$$
\frac{1}{\left(1-2 x z+z^{2}\right)^{n+1 / 2}}=\sum_{k=0}^{\infty} C_{k}^{n+1 / 2}(x) z^{k}
$$

Comparing this with the expansion

$$
G(w, z, t)=\sum_{k=0}^{\infty} \sum_{m=0}^{\infty} \frac{w^{m}}{m !} z^{k+m+1 / 2}\left\langle\tilde{k}\left|\hat{a}^{m} \hat{U}_{\operatorname{lin}}^{t}\right| \tilde{k}\right\rangle
$$

obtained by evaluating the trace $\mathrm{A} 1 \mathrm{l}$ ) in an eigenbasis of $\hat{h}$ leads us to the result

$$
\left\langle\tilde{k}\left|\hat{a}^{2 n} \hat{U}_{\operatorname{lin}}^{t}\right| \tilde{k}\right\rangle=\frac{(2 n) !}{2^{n} n !} \sin ^{n} \psi \sqrt{\cos \psi} C_{k}^{n+1 / 2}(\cos \psi) e^{i(k+1 / 2)(\phi-\mu t \pi)+i n \phi+i n \pi / 2}
$$

from which we determine $A_{k+2 n, k}^{\mathrm{lin}}(t)$ as given in (13). Note that we find $A_{l k}^{\mathrm{lin}}(t)=0$ unless $l$ and $k$ are both even or both odd. This is because the linearised system enjoys an inversion symmetry even when the original system does not. 


\section{APPENDIX B: TRACING THE AFFINE TRANSFORMATION}

In this appendix we derive (A3) using the trace formula [22]. We consider first the general affine transformation

$$
\zeta \mapsto M \zeta+Z
$$

where $M$ and $Z=(a, b)$ are an arbitrary symplectic matrix and phase-space displacement respectively. For convenience we may initially suppose that $M$ and $Z$ are real and generalise to complex transformations later using analytic continuation.

The transformation in (B1) has a single fixed point

$$
\zeta=-(M-I)^{-1} Z
$$

The trace formula then gives [22]

$$
\operatorname{Tr} \hat{T}(Z) \hat{U}(M)=\frac{e^{i S_{0} / \hbar}}{\sqrt{\operatorname{Tr} M-2}}
$$

where $\hat{T}(Z)$ and $\hat{U}(M)$ are the operators which quantise $\zeta \mapsto \zeta+Z$ and $\zeta \mapsto M \zeta$ respectively and $S_{0}$ is the action of the fixed point. By evaluating the generating function for $M$ at the fixed point one can show that

$$
\begin{aligned}
S_{0} & =\frac{1}{2}(b q-a p) \\
& =\frac{1}{2} \Omega(\zeta, Z) \\
& =\frac{1}{2} \Omega\left(Z,(M-I)^{-1} Z\right),
\end{aligned}
$$

where $\Omega(u, v)=u^{T} J v$ is the symplectic form and $\zeta=(q, p)$.

We specialise to the trace in (A1) by substituting the $\delta \zeta$ defined in (A2) for $Z$ and $W(z) M_{0}^{t}$ for $M$, giving

$$
G(w, z, t)=\frac{e^{i w^{2} \Xi(z, t) / 4}}{\sqrt{\operatorname{Tr} W(z) M_{0}^{t}-2}},
$$

where

$$
\Xi(z, t)=\Omega\left(\zeta_{c}, \frac{1}{W(z) M_{0}^{t}-I} \zeta_{c}\right) \quad \text { and } \quad \zeta_{c}=\left(\begin{array}{c}
1 \\
i
\end{array}\right)
$$

Notice that, because $\delta \zeta$ scales as $\hbar^{1 / 2}$, there is no $\hbar$-dependence in the final result.

We are free to work in a coordinate system in which $K=I$ and then

$$
W(z)=e^{i \ln z J}=z(I+i J)+\frac{1}{z}(I-i J) .
$$

Consider first the case

$$
M_{0}^{t}=(-1)^{\mu t}\left(\begin{array}{cc}
\cosh \rho t & e^{\sigma} \sinh \rho t \\
e^{-\sigma} \sinh \rho t & \cosh \rho t
\end{array}\right) .
$$

where $\sigma$ and $\rho$ are constants. Substitution in (B2) gives then

$$
\Xi(z, t)=2(-1)^{\mu t} \cosh \sigma \sinh \rho t \frac{z}{\Delta(z, t)},
$$


where $\Delta(z, t)$ was defined in appendix A. Explicit calculation of $\Delta(z, t)$ for this $M_{0}$ gives

$$
Q=-\sinh \sigma .
$$

Using the identity

$$
\sec ^{2} \psi(t)=\cosh ^{2} \rho t+\sinh ^{2} \sigma \sinh ^{2} \rho t=1+\cosh ^{2} \sigma \sinh ^{2} \rho t
$$

we then get $\tan \psi(t)=\cosh \sigma \sinh \rho t$ and hence (A3).

Any hyperbolic matrix can be put in the form (B3) following an orthogonal transformation which rotates coordinates by an angle $\theta_{0}$. The matrix $W(z)$ is unchanged by such a transformation. However, the annihilation operator is multiplied by a phase

$$
\hat{a} \rightarrow e^{i \theta_{0}} \hat{a}
$$

and the exponent of the generating function by

$$
\Xi(z, t) \rightarrow e^{2 i \theta_{0}} \Xi(z, t) .
$$

In systems with time-reversal symmetry we can demand that the eigenvectors $|\tilde{k}\rangle$ be real and this allows us to fix the possible rotation angles $\theta_{0}$ to multiples of $\pi / 2$. Among these, only $\theta_{0}=0$ and $\theta_{0}=\pi / 2$ give distinct results for $\Xi(z, t)$. The formula we have given applies to the case $\theta_{0}=0$. In the case $\theta_{0}=\pi / 2$, the formula holds as given if we rephase the eigenvectors according to $|\tilde{k}\rangle \rightarrow(-1)^{k}|\tilde{k}\rangle$.

We can similarly use a convention for $|\tilde{k}\rangle$ such that $(13)$ holds in the GUE case. This amounts in particular to adopting a convention in which the covariance matrix $C_{l k}(E)=\left\langle x_{l} x_{k}^{*}\right\rangle$ is real even though individual values of $x_{l} x_{k}^{*}$ are complex. This reflects the fact that even in systems without global time-reversal symmetry, the linearised dynamics from which $A_{l k}^{\operatorname{lin}}(t)$ is calculated is time-reversal invariant and we are free to choose the eigenstates $|\tilde{k}\rangle$ in a way which reflects this. This is in direct analogy to the spatial symmetry mentioned in the previous appendix which the linearised dynamics also possesses even in the absence of an equivalent symmetry for the global dynamics. 